\title{
Expression and diagnostic value of miR-34c and miR-141 in serum of patients with colon cancer
}

\author{
HUIJING WU ${ }^{1}$ and HONGXIA YAN ${ }^{2}$ \\ ${ }^{1}$ Department of Medical Oncology, Radio-Chemotherapy Center; \\ ${ }^{2}$ Department of Oncological Radiotherapy, Hubei Cancer Hospital, Wuhan, Hubei 430079, P.R. China
}

Received October 16, 2019; Accepted July 21, 2020

DOI: 10.3892/ol.2020.11959

\begin{abstract}
Expression of miR-34c and miR-141 in serum of colon cancer patients and their association with clinicopathological features and diagnostic value for colon cancer were investigated. A total of 64 patients with colon cancer admitted to Hubei Cancer Hospital from January 2016 to March 2018 were included in the experimental group, and 64 healthy subjects undergoing physical examination during the same period were the control group. The expression of miR-34c and miR-141 in serum of patients in the two groups were detected by RT-qPCR, and the association of miR-34c and miR-141 with the clinicopathological characteristics of colon cancer patients was analyzed. The receiver operating characteristic (ROC) curve was used to assess the diagnostic efficiency of miR-34c and miR-141 in colon cancer. The expression of miR-141 in serum of patients in the experimental group was significantly higher than that in the control group $(\mathrm{P}<0.05)$. Expression of miR-34c in serum of patients in the experimental group was significantly lower than that in the control group $(\mathrm{P}<0.05)$ and the expression of miR-34c and miR-141 in serum of the experimental group were associated with tumor diameter, clinical stage, degree of differentiation and lymph node metastasis $(\mathrm{P}<0.05)$. AUC of serum miR-34c in the diagnosis of colon cancer was 0.857 (95\% CI: 0.795-0.919), with the cut-off value of 0.800, the diagnostic sensitivity of $84.38 \%$, and the specificity of $68.75 \%$ and AUC of serum miR-141 in the diagnosis of colon cancer was 0.876 (95\% CI: 0.810-0.941), with the cut-off value of 0.282 , the diagnostic sensitivity of $70.31 \%$, and the specificity of $96.88 \%$. The ROC curve for the diagnosis of colon cancer was further plotted in combination with serum miR-34c and miR-141. AUC of the two combined for the diagnosis of colon
\end{abstract}

Correspondence to: Dr Hongxia Yan, Department of Oncological Radiotherapy, Hubei Cancer Hospital, 116 Zhuodao Quan'nan Road, Hongshan, Wuhan, Hubei 430079, P.R. China

E-mail: yc736j@163.com

Key words: miR-34c, miR-141, colon cancer, clinical pathology, diagnostic value cancer was 0.929 (95\% CI: $0.884-0.974)$, with the cut-off value of 0.566 , the diagnostic sensitivity of $84.38 \%$, and the specificity of $93.75 \%$. In conclusion, miR-34c and miR-141 might be involved in the occurrence and progression of colon cancer and could be used as biological indicators for early diagnosis of colon cancer.

\section{Introduction}

Colon cancer is one of the most common malignant tumors in the clinical practice. Its morbidity and mortality worldwide are gradually increasing, posing a great threat to human life (1). There is lack of specific early stage symptoms and the digestive system signs appear in the middle and late stages, which can cause serious adverse effects $(2,3)$. At present, the main treatment for clinical colon cancer is still surgical resection, but due to the lack of sensitive diagnostic indicators, most patients have already developed lymph nodes or distant metastases at the time of diagnosis, and the surgical resection rate and prognosis are not ideal $(4,5)$. Therefore, it is very important to find a biomarker for early diagnosis of colon cancer.

It has been found that miRNAs are differentially expressed in colon cancer cells (6). They are closely related to the biological and clinical characteristics of colon cancer, and play an important role in the occurrence and progression of colon cancer (7). As studies have shown, miRNAs are thought to have a regulator role in tumor suppression and tumorigenesis. For example, miR-185 can inhibit the proliferation and invasion of colon cancer cells by targeting Wnt1, and regulating the level of miR-185 may have a therapeutic effect on colon cancer patients (8). miR-223-3p can promote the progression of colon cancer by negatively regulating PRDM1 (9). Based on these results, miRNAs are attracting attention as a potential target for the treatment and diagnosis of colon cancer. Recently, the role of miR-141 in tumor growth has been described. Expression level of miR-141 in patients with colon cancer at stage IV has increased, which can easily distinguish patients with distant metastasis, other stages and healthy control, showing that plasma miR-141 is a potential prognostic factor for predicting poor survival of colon cancer patients (10). miR-34C is a tumor suppressor regulator, which is down-regulated in most forms of cancer, and can inhibit the growth of malignant tumors by inhibiting genes related to proliferation, anti-apoptosis 
and migration (11). A study has shown that the expression of miR-34C is down-regulated in colon cancer, and the loss of expression is consistent with the data of colon cancer cell lines (12).

There are few previous studies on the diagnosis of colon cancer with serum miR-34c and miR-141 (13-15), and the study on the role of miRNA expression in colon cancer provided new diagnostic methods and thinking for the diagnosis and treatment of colon cancer. In this study, by observing the expression of miR-34c and miR-141 in the serum of colon cancer patients, the diagnostic value of miR-34c and miR-141 in colon cancer and their relationship with clinicopathological features were investigated.

\section{Patients and methods}

General data. A total of 64 patients diagnosed with colon cancer and treated in Hubei Cancer Hospital (Wuhan, China) were selected as the experimental group, including 44 males and 20 females. The patients were aged 25-65 years, with an average age of $(46.5 \pm 8.4)$ years. According to TNM staging system, there were 22 cases of stage I+II, and 42 cases of stage III. There were 19 cases of lymph node metastasis, and 15 cases of poor differentiation, 49 cases of high and medium differentiation. Sixty-four healthy subjects were included in the control group, including 38 males and 26 females. The controls were aged 26-57 years, with an average age of $(44.5 \pm 7.9)$ years. The study was approved by the Ethics Committee of the Hubei Cancer Hospital, and the subjects and/or their families were informed and signed an informed consent.

Inclusion and exclusion criteria. Inclusion criteria were: Patients met NCCN colon cancer tumor clinical practice guidelines (16). CT, color Doppler ultrasound and MRI were performed to rule out distant metastasis. According to TNM staging system there were Stage I, II and III. Patients did not receive previous chemotherapy, or radiotherapy. Patients were diagnosed for the first time, with detailed clinicopathological data. Exclusion criteria were: Patients without other malignant tumors, hematological diseases. Patients with severe complications and immune system diseases. Patients with poor treatment compliance caused by severe mental illness, and patients unwilling to participate in the present study.

Main instruments and reagents. ABI PRISM 7500 quantitative PCR instrument (Beijing Image Trading Co., Ltd.; cat. no. 100005). M-MLV reverse transcription kit (Beijing Shengkeboyuan Biotechnology Co., Ltd.; cat. no. RTP50). TRIzol extraction kit (Shanghai Xinfan Biological Technology Co., Ltd.; cat. no. XFR1030). UV-visible spectrophotometer (Bioteke corporation; cat. no. ND5000). microRNA PCR premix kit (AcebioX; cat. no. PAMI000). The primers of miR-34c, miR-141 and U6 were synthesized by Beijing Lvyuanbode Biotechnology Co., Ltd. (Table I).

$R T$-qPCR detection. Elbow venous blood $(5 \mathrm{ml})$ of the subjects were taken, after 10-15 $\mathrm{min}$, the blood was centrifuged at $1,500 \mathrm{x} \mathrm{g}$ and $4^{\circ} \mathrm{C}$ for $10 \mathrm{~min}$, and then stored at $-70^{\circ} \mathrm{C}$. The total RNA in the serum was extracted using TRIzol kit (Takara), and the absorbance values of RNA at 260 and $280 \mathrm{~nm}$ were measured by ultraviolet-visible spectrophotometer. The RNA concentration and purity were analyzed. Then, $2 \mu 1$ of total RNA was taken to reversely transcribe the first strand cDNA, according to the reverse transcription kit. Reverse transcription reaction conditions: $42^{\circ} \mathrm{C}$ for $30 \mathrm{~min}, 95^{\circ} \mathrm{C}$ for $5 \mathrm{~min}$. The synthesized cDNA sample was stored at $-80^{\circ} \mathrm{C}$. U6 was used as an internal reference. The total volume of $20 \mu \mathrm{l}$ includes $10 \mu \mathrm{l}$ of PCR Premix, $2 \mu \mathrm{l}$ of upstream primer, $2 \mu \mathrm{l}$ of downstream primer and dd water (Rnase and Dnase free). PCR amplification cycle conditions were: $92^{\circ} \mathrm{C}$ for $5 \mathrm{~min}, 95^{\circ} \mathrm{C}$ for $5 \mathrm{sec}, 65^{\circ} \mathrm{C}$ for $30 \mathrm{sec}, 72^{\circ} \mathrm{C}$ for $5 \mathrm{sec}$, a total of 45 cycles. $2^{-\Delta \Delta \mathrm{Cq}}$ method was used to analyze the relative expression of the target gene.

Statistical analysis. SPSS19.0 (IBM Corp.) statistical software was used to analyze the data. Enumeration data in the group were represented by numbers of case/percentage [n (\%)] and were analyzed by Chi-square test. Measurement data were expressed by mean \pm standard deviation. Independent sample t-test was used for comparison of measurement data between two groups. ROC curve was used to assess the diagnostic efficiency of miR-34c and miR-141 on colon cancer. $\mathrm{P}<0.05$, was considered statistically significant.

\section{Results}

General data. There was no difference between the experimental group and the control group in gender, age, body mass index (BMI), smoking history, drinking history, residence, educational level or other general clinical data (P>0.05) (Table II).

Expression levels of miR-34c and miR-141 in colon cancer. The relative expression of miR-34c and miR-141 in the serum of subjects in the two groups were detected, and it was found that the serum level of miR-34c in the experimental group was significantly lower than that in the control group $(\mathrm{P}<0.05)$, and the relative expression of miR-141 in serum of patients in the experimental group was significantly higher than that in the control group $(\mathrm{P}<0.05)$ (Table III and Fig. 1).

Association of the expression of $m i R-34 c$ and $m i R-141$ with clinicopathologic features of patients with colon cancer. The expression of miR-34c and miR-141 in serum of colon cancer was not associated with the clinicopathological parameters such as age, gender, local tumor invasion, vascular invasion, degree of differentiation, and neural invasion $(\mathrm{P}>0.05)$, but was associated with tumor diameter, lymph node metastasis, carcinoembryonic antigen, and TNM staging $(\mathrm{P}<0.05)$ (Table IV).

The relative expression of serum $\mathrm{miR}-34 \mathrm{c}$ and $\mathrm{miR}-141$ in the diagnosis efficiency of colon cancer. ROC curve of relative expression of serum miR-34c and miR-141 for the diagnosis of colon cancer was drawn. AUC of serum miR-34c for the diagnosis of colon cancer was 0.857 (95\% CI: 0.795-0.919), its cut-off value was 0.800 ; diagnostic sensitivity was $84.38 \%$, and specificity was $68.75 \%$. AUC of serum miR-141 for 
Table I. Primer sequences of miR-34c, miR-141 and U6.

\begin{tabular}{lll}
\hline Gene & \multicolumn{1}{c}{ Forward primers } & \multicolumn{1}{c}{ Reverse primers } \\
\hline miR-34c & 5'-CGCGGATCCTCTATTTGCCATCGTCTA-3' & 5'-CTGAAGCTTCAGGCAGCTCATTTGGAC-3' \\
miR-141 & 5'-GCGAAGCATTTGCCAAGAA-3' & 5'-CAATCACAGACCTGTTATTGC-3' \\
U6 & 5'-CTCGCTTCGGCAGCACA-3' & 5'-AACGCTTCACGAATTTGCGT-3' \\
\hline
\end{tabular}

Table II. General data in the experimental group and control group [n (\%), mean \pm SD].

\begin{tabular}{|c|c|c|c|c|}
\hline Class & Experimental group $(n=64)$ & Control group $(n=64)$ & $\mathrm{t} / \chi^{2}$ & P-value \\
\hline Sex & & & 0.131 & 0.717 \\
\hline Male & $44(68.75)$ & $38(59.38)$ & & \\
\hline Female & $20(31.25)$ & $26(40.62)$ & & \\
\hline Age (years) & $46.5 \pm 8.4$ & $44.5 \pm 7.9$ & 1.388 & 0.168 \\
\hline BMI $\left(\mathrm{kg} / \mathrm{m}^{2}\right)$ & $22.94 \pm 4.06$ & $22.49 \pm 3.72$ & 0.654 & 0.514 \\
\hline Smoking history & & & 0.283 & 0.595 \\
\hline Yes & $36(56.25)$ & $33(51.56)$ & & \\
\hline No & $28(43.75)$ & $31(48.44)$ & & \\
\hline Drinking history & & & 1.647 & 0.199 \\
\hline Yes & $44(68.75)$ & $37(57.81)$ & & \\
\hline No & $20(31.25)$ & $27(42.19)$ & & \\
\hline Place of residence & & & 0.142 & 0.707 \\
\hline City & $20(31.25)$ & $22(34.38)$ & & \\
\hline Country & $44(68.75)$ & $42(65.62)$ & & \\
\hline Education level & & & 2.050 & 0.152 \\
\hline$>$ Senior high school & $33(51.56)$ & $41(64.06)$ & & \\
\hline$\leq$ Senior high school & $31(48.44)$ & $23(35.94)$ & & \\
\hline
\end{tabular}
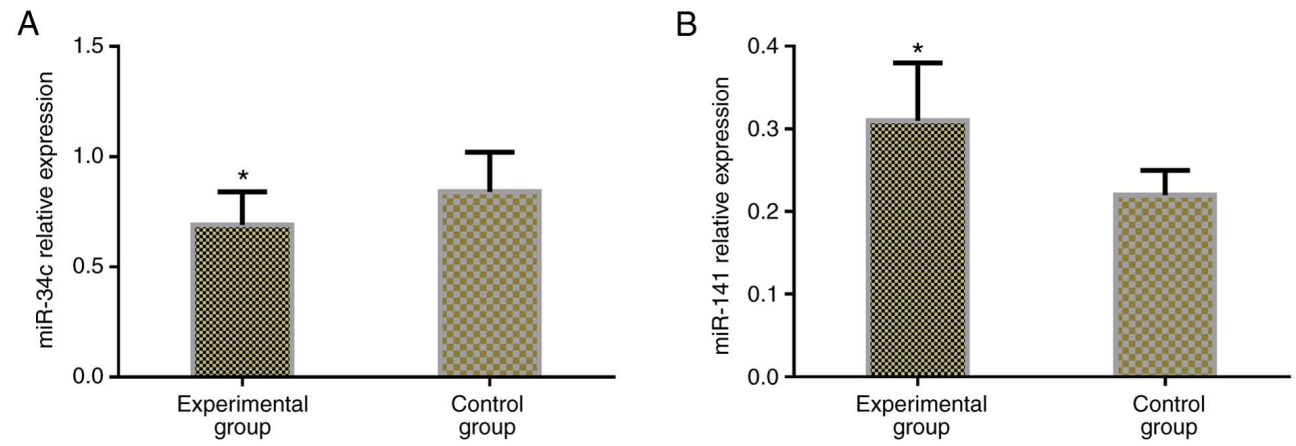

Figure 1. Expression of miR-34c and miR-141 in the serum of colon cancer. (A) Serum level of miR-34c in the experimental group was significantly lower than that in the control group $(\mathrm{P}<0.05)$. (B) The relative expression of miR-141 in serum of patinets in the experimental group was significantly higher than that of the control group $(\mathrm{P}<0.05) .{ }^{*} \mathrm{P}<0.05$, compared with the normal control group.

diagnosis of colon cancer was 0.876 (95\% CI: 0.810-0.941), its cut-off value was 0.282 ; diagnostic sensitivity was $70.31 \%$, and specificity was $96.88 \%$. ROC curve for the diagnosis of colon cancer combined with serum miR-34c and miR-141 was further drawn. The combined AUC of serum miR-34c and miR-141 for the diagnosis of colon cancer was 0.929 (95\% CI: $0.884 \sim 0.974$ ), the cut-off value was 0.566 ; diagnosis sensitivity was $84.38 \%$ and the specificity was $93.75 \%$. More details are shown in Table V and Fig. 2.
Table III. Expression levels of miR-34c and miR-141 in serum of colon cancer (mean $\pm \mathrm{SD}$ ).

\begin{tabular}{lccc}
\hline Groups & $\mathrm{n}$ & $\mathrm{miR}-34 \mathrm{c}$ & $\mathrm{miR}-141$ \\
\hline Experimental group & 64 & $0.69 \pm 0.15$ & $0.31 \pm 0.07$ \\
Control group & 64 & $0.84 \pm 0.18$ & $0.22 \pm 0.03$ \\
t & - & 5.121 & 9.454 \\
P-value & - & $<0.001$ & $<0.001$ \\
\hline
\end{tabular}


Table IV. Correlation of miR-34c and miR-141 expression levels with the clinicopathologic features of patients with colon cancer $($ mean $\pm \mathrm{SD})$.

\begin{tabular}{|c|c|c|c|c|c|c|c|}
\hline Clinicopathologic features & $\mathrm{n}$ & $\operatorname{miR}-34 c(n=64)$ & $\mathrm{t}$ & P-value & $\operatorname{miR}-141(\mathrm{n}=64)$ & $\mathrm{t}$ & P-value \\
\hline Sex & & & 1.577 & 0.120 & & 1.783 & 0.079 \\
\hline Male & 44 & $0.65 \pm 0.12$ & & & $0.32 \pm 0.08$ & & \\
\hline Female & 20 & $0.71 \pm 0.18$ & & & $0.28 \pm 0.09$ & & \\
\hline Age, years & & & 0.712 & 0.479 & & 1.445 & 0.154 \\
\hline$<50$ & 21 & $0.67 \pm 0.13$ & & & $0.30 \pm 0.09$ & & \\
\hline$\geq 50$ & 43 & $0.70 \pm 0.17$ & & & $0.33 \pm 0.11$ & & \\
\hline Tumor diameter, $\mathrm{cm}$ & & & 3.177 & 0.002 & & 1.612 & 0.112 \\
\hline$<5$ & 47 & $0.74 \pm 0.13$ & & & $0.33 \pm 0.12$ & & \\
\hline$\geq 5$ & 17 & $0.61 \pm 0.18$ & & & $0.33 \pm 0.16$ & & \\
\hline TNM stage & & & 4.512 & $<0.001$ & & 3.438 & 0.018 \\
\hline Grade I+II & 22 & $0.81 \pm 0.18$ & & & $0.29 \pm 0.05$ & & \\
\hline Grade III & 42 & $0.64 \pm 0.12$ & & & $0.34 \pm 0.09$ & & \\
\hline Grade of differentiation & & & 1.862 & 0.673 & & 0.904 & 0.369 \\
\hline High and moderate differentiation & 49 & $0.70 \pm 0.12$ & & & $0.32 \pm 0.07$ & & \\
\hline Poor differentiation & 15 & $0.63 \pm 0.15$ & & & $0.30 \pm 0.09$ & & \\
\hline Lymph node metastasis & & & 8.204 & $<0.001$ & & 2.318 & 0.024 \\
\hline With & 19 & $0.43 \pm 0.12$ & & & $0.34 \pm 0.07$ & & \\
\hline Without & 45 & $0.80 \pm 0.18$ & & & $0.30 \pm 0.06$ & & \\
\hline Local tumor invasion & & & 1.842 & 0.703 & & 1.592 & 0.116 \\
\hline With & 33 & $0.71 \pm 0.17$ & & & $0.33 \pm 0.08$ & & \\
\hline Without & 31 & $0.64 \pm 0.13$ & & & $0.30 \pm 0.07$ & & \\
\hline Vascular invasion & & & 0.533 & 0.596 & & 1.500 & 0.139 \\
\hline With & 35 & $0.68 \pm 0.14$ & & & $0.33 \pm 0.04$ & & \\
\hline Without & 29 & $0.70 \pm 0.16$ & & & $0.30 \pm 0.11$ & & \\
\hline Neural invasion & & & 1.033 & 0.306 & & 1.013 & 0.315 \\
\hline With & 40 & $0.67 \pm 0.15$ & & & $0.34 \pm 0.08$ & & \\
\hline Without & 24 & $0.71 \pm 0.15$ & & & $0.32 \pm 0.07$ & & \\
\hline Carcinoembryonic antigen, $\mu \mathrm{g} / 1$ & & & 3.968 & 0.002 & & 2.667 & 0.009 \\
\hline$\geq 3.4$ & 34 & $0.61 \pm 0.17$ & & & $0.34 \pm 0.07$ & & \\
\hline$<3.4$ & 30 & $0.77 \pm 0.15$ & & & $0.29 \pm 0.08$ & & \\
\hline
\end{tabular}

Table V. Relative expression of serum miR-34c and miR-141 in the diagnostic efficiency of colon cancer.

\begin{tabular}{lcccccc}
\hline Diagnostic indicator & AUC & $95 \%$ CI & Standard error & Cut-off value & Sensitivity (\%) & Specificity (\%) \\
\hline miR-34c & 0.857 & $0.795-0.919$ & 0.032 & 0.800 & 84.38 & 68.75 \\
miR-141 & 0.876 & $0.810-0.941$ & 0.033 & 0.282 & 70.31 & 96.88 \\
miR-34c+miR-141 & 0.929 & $0.884-0.974$ & 0.023 & 0.566 & 84.38 & 93.75 \\
\hline
\end{tabular}

\section{Discussion}

Colon cancer is a malignant lesion in mucosal epithelium caused by a variety of carcinogenic factors. It is mainly related to a high-fat, high-protein and low-fiber diet, and is one of the common malignant tumors (17). The onset of colon cancer is insidious and most of its onset is slow. In the early stage, there is no obvious symptoms, patients are often diagnosed in the middle or late stage, thus losing the best time for treatment (18). Therefore, how to improve the diagnostic rate of colon cancer and overall survival is a major problem for clinicians. Surgery is an early treatment method for colon cancer. In recent years, the treatment of colon cancer has improved to a certain extent, but the high postoperative complication rate of colon cancer has not changed (19). Hence, actively looking for indicators with high sensitivity is 


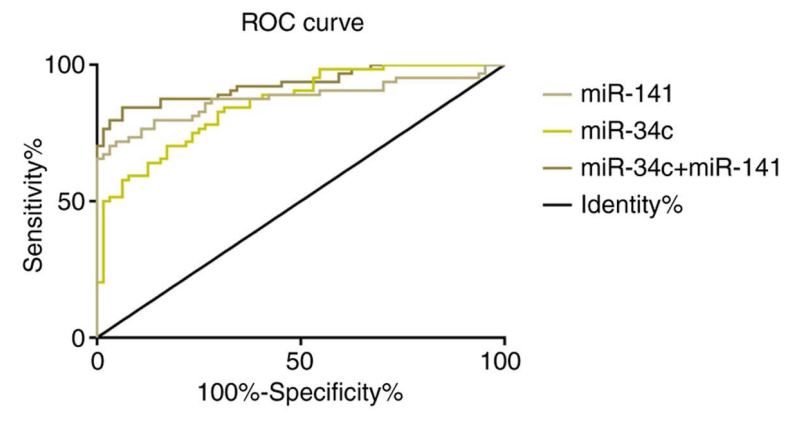

Figure 2. The relative expression of serum miR-34c and miR-141 in the diagnosis of colon cancer. The AUC of serum miR-34c for the diagnosis of colon cancer was 0.857 (95\% CI: 0.795-0.919), its cut-off value was 0.800 , diagnostic sensitivity was $84.38 \%$, and specificity was $68.75 \%$. AUC of serum miR-141 for diagnosis of colon cancer was 0.876 (95\% CI: 0.810-0.941), its cut-off value was 0.282 , diagnostic sensitivity was $70.31 \%$, and specificity was $96.88 \%$. ROC curve for the diagnosis of colon cancer combined with serum miR-34c and miR-141 was drawn, the combined AUC of serum miR-34c and miR-141 for the diagnosis of colon cancer was $0.929(95 \% \mathrm{CI}$ : $0.884-0.974$ ), the cut-off value was 0.566 , diagnosis sensitivity was $84.38 \%$ and the specificity was $93.75 \%$

of great significance for the early diagnosis and improvement of prognosis of colon cancer.

Colonoscopy is the current choice for clinical screening of colon cancer $(20,21)$. Due to its high cost and invasive characteristics, colonoscopy is not widely used in clinical practice. The other choice, fecal occult blood test, has extremely high dietary requirements for patients due to its low sensitivity (22), so invasive biomarkers are urgently needed to detect colon cancer. miRNAs can form specific inclusion bodies and exosomes, which are suitable for detection in body fluids, serum and feces (23), making it possible to detect miRNAs in serum as biomarkers for nasopharyngeal cancer.

Micro non-coding microRNAs (miRNAs) contribute to the development and progression of cancer and are differentially expressed in normal tissues and cancer (24). miRNAs have a 'dual identity' of oncogene and cancer suppressor gene in the process of tumor progression, which is closely related to the occurrence, progression and metastasis of tumors (25). By regulating different signaling pathways, miR-141 indirectly regulates physiological and pathological conditions and plays an important role in the progression of the disease. It has been shown to be low expressed in a variety of tumors, and to be significantly decreased in tumor tissues, lymph nodes, and sera of colon cancer patients. Studies have revealed that the expression of miRNA-141 is down-regulated in colon cancer, which improves the expression level of MAP4K4, changes the anti-tumor response, and further increases tumor proliferation, invasion and metastasis (26). miR-34c is down-regulated in many different malignancies. Previous studies have revealed that the down-regulation of miR-34c in endometrial cancer may be an important factor for poor prognosis. Its overexpression can inhibit cell proliferation, colony formation, invasion, metastasis and apoptosis, and plays an important role in the formation, occurrence and progression of a variety of tumors (27). For example, in the study of Yang et al (28), the silencing of tumor suppressors such as miR-34c was related to the development of colorectal cancer, and showed that the overexpression of miR-34c induced apoptosis by silencing its target cytokines and inhibited the invasion and proliferation of colorectal cancer cells, indicating that miR-34c was a promising target. In the study of Gao et al (29), the dysregulation of miR-141 depended on the type of cancer. It played a dual role in tumorigenicity, and regulated cell movement and controlling 'dryness'. This phenomenon strongly suggested that miR-141 was an oncogene or tumor suppressor gene and provided new options for targeting cancer therapies. In this study, the relative expression of miR-34c and miR-141 in serum of patients in the two groups were detected. The serum level of miR-34c in the experimental group was significantly lower than that in the control group, while the expression of miR-141 was significantly higher than that of the control group, and was associated with tumor diameter, carcinoembryonic antigen, lymph node metastasis and TNM staging $(\mathrm{P}<0.05)$, indicating that miR-34c and miR-141 might be involved in the occurrence and progression of colon cancer, and miR-34c might act as a tumor suppressor gene in colon cancer, and miR-141 might act as an oncogene.

It has been reported that miR-34c and miR-141 can be used as biomarkers in a variety of tumors. For example, in the study of Tao et al (30), miR-34c played a key role in ovarian cancer and could be used as a potential diagnostic biomarker and a powerful therapeutic target for ovarian cancer. Wang et al (31) reported that the overexpression of miR-141 was not only closely related to the classification and size of osteosarcoma, but also inhibited the growth and metastasis of osteosarcoma cells by regulating epidermal growth factor and affecting downstream pathway proteins. The results of this study showed that the sensitivity and specificity of serum miR-34c for the diagnosis of colon cancer were 84.38 and $68.75 \%$, respectively, and the sensitivity and specificity of serum miR-141 for the diagnosis of colon cancer were 70.31 and $96.88 \%$, respectively. The sensitivity and specificity of the combined diagnosis of colon cancer were 84.38 and $93.75 \%$, respectively. It suggested that miR-34c and miR-141 could be used as biological indicators with good sensitivity and specificity in the diagnosis of colon cancer, and their combination could improve the sensitivity in the diagnosis of colon cancer. Therefore, miR-34c and miR-141 might play important roles in the occurrence, progression and prognosis of colon cancer.

The research subjects were screened strictly in accordance with the inclusion and exclusion criteria, and there were no significant differences between the experimental group and the control group in the general clinical baseline data of gender and age, which ensured the preciseness and reliability of the study. Although this study confirmed the role of miR-34c and miR-141 in the occurrence, progression and prognosis of colon cancer, the functions of miR-34c and miR-141 in the prognosis of patients were not observed. These deficiencies needed to be further supplemented in future studies to support the results of this study.

In conclusion, miR-34c and miR-141 might be involved in the occurrence and progression of colon cancer. Serum miR-34c and miR-141 were detected to have a good sensitivity and specificity in the diagnosis of colon cancer, and combined detection could improve the sensitivity in the diagnosis of colon cancer. The combination of the two might be a new biomarker for colon cancer diagnosis. 


\section{Acknowledgements}

Not applicable.

\section{Funding}

No funding was received.

\section{Availability of data and materials}

The datasets used and/or analyzed during the present study are available from the corresponding author on reasonable request.

\section{Authors' contributions}

HW and HY conceived and designed the study, acquired, analyzed and interpreted the experiment data, drafted the manuscript, and revised the manuscript critically for important intellectual content. Both authors read and approved the final manuscript.

\section{Ethics approval and consent to participate}

The study was approved by the Ethics Committee of Hubei Cancer Hospital (Wuhan, China). Signed informed consents were obtained from the patients and/or guardians.

\section{Patient consent for publication}

Not applicable.

\section{Competing interests}

The authors declare that they have no competing interests.

\section{References}

1. Nakagawa-Senda H, Hori M, Matsuda T and Ito H: Prognostic impact of tumor location in colon cancer: The Monitoring of Cancer Incidence in Japan (MCIJ) project. BMC Cancer 19: 431, 2019.

2. Zhu J, Dong H, Zhang Q and Zhang S: Combined assays for serum carcinoembryonic antigen and microRNA-17-3p offer improved diagnostic potential for stage I/II colon cancer Mol Clin Oncol 3: 1315-1318, 2015.

3. Paudel MS, Mandal AK, Shrestha B, Poudyal NS, Kc S, Chaudhary S, Shrestha R and Goel K: Prevalence of organic colonic lesions by colonoscopy in patients fulfilling ROME IV criteria of irritable bowel syndrome. JNMA J Nepal Med Assoc 56: 487-492, 2018.

4. Mayanagi S, Kashiwabara K, Honda M, Oba K, Aoyama T, Kanda M, Maeda H, Hamada C, Sadahiro S, Sakamoto J, et al: Risk factors for peritoneal recurrence in stage II to III colon cancer. Dis Colon Rectum 61: 803-808, 2018.

5. Zhang Q, Zhang C, Ma JX, Ren H, Sun Y and Xu JZ: Circular RNA PIP5K1A promotes colon cancer development through inhibiting miR-1273a. World J Gastroenterol 25: 5300-5309, 2019.

6. Brase JC, Wuttig D, Kuner R and Sültmann H: Serum microRNAs as non-invasive biomarkers for cancer. Mol Cancer 9: 306, 2010.

7. Zeng M, Zhu L, Li L and Kang C: miR-378 suppresses the proliferation, migration and invasion of colon cancer cells by inhibiting SDAD1. Cell Mol Biol Lett 22: 12, 2017.

8. Sheng S, Xie L, Wu Y, Ding M, Zhang T and Wang X: MiR-144 inhibits growth and metastasis in colon cancer by down-regulating SMAD4. Biosci Rep 39: BSR20181895, 2019.
9. Zhang W, Sun Z, Su L, Wang F, Jiang Y, Yu D, Zhang F, Sun Z and Liang W: miRNA-185 serves as a prognostic factor and suppresses migration and invasion through Wnt1 in colon cancer. Eur J Pharmacol 825: 75-84, 2018.

10. Chai B, Guo Y, Cui X, Liu J, Suo Y, Dou Z and Li N: MiR-223-3p promotes the proliferation, invasion and migration of colon cancer cells by negative regulating PRDM1. Am J Transl Res 11: 4516-4523, 2019.

11. Cheng H, Zhang L, Cogdell DE, Zheng H, Schetter AJ, Nykter M, Harris CC, Chen K, Hamilton SR and Zhang W: Circulating plasma MiR-141 is a novel biomarker for metastatic colon cancer and predicts poor prognosis. PLoS One 6: e17745, 2011.

12. Hagman Z, Haflidadottir BS, Ansari M, Persson M, Bjartell A, Edsjö A and Ceder Y: The tumour suppressor miR-34c targets MET in prostate cancer cells. Br J Cancer 109: 1271-1278, 2013.

13. Roy S, Levi E, Majumdar AP and Sarkar FH: Expression of miR-34 is lost in colon cancer which can be re-expressed by a novel agent CDF. J Hematol Oncol 5: 58, 2012.

14. Liang Z, Li X, Liu S, Li C, Wang X and Xing J: MiR-141-3p inhibits cell proliferation, migration and invasion by targeting TRAF5 in colorectal cancer. Biochem Biophys Res Commun 514: 699-705, 2019.

15. Wan Y, Shen A, Qi F, Chu J, Cai Q, Sferra TJ, Peng J and Chen Y: Pien Tze Huang inhibits the proliferation of colorectal cancer cells by increasing the expression of miR-34c-5p. Exp Ther Med 14: 3901-3907, 2017.

16. Benson AB III, Venook AP, Cederquist L, Chan E, Chen YJ, Cooper HS, Deming D, Engstrom PF, Enzinger PC, Fichera A, et al: Colon cancer, version 1.2017, NCCN clinical practice guidelines in oncology. J Natl Compr Canc Netw 15: 370-398, 2017.

17. Schweiger MR, Hussong M, Röhr $\mathrm{C}$ and Lehrach $\mathrm{H}$ : Genomics and epigenomics of colorectal cancer. Wiley Interdiscip Rev Syst Biol Med 5: 205-219, 2013.

18. Soler M, Estevez MC, Villar-Vazquez R, Casal JI and Lechuga LM: Label-free nanoplasmonic sensing of tumor-associate autoantibodies for early diagnosis of colorectal cancer. Anal Chim Act 930: 31-38, 2016.

19. Arnarson Ö, Butt-Tuna S and Syk I: Postoperative complication following colonic resection for cancer is associated with impaired long-term survival. Colorectal Dis 21: 805-815, 2019.

20. Baxter NN, Goldwasser MA, Paszat LF, Saskin R, Urbach DR and Rabeneck L: Association of colonoscopy and death from colorectal cancer. Ann Intern Med 150: 1-8, 2009.

21. Lieberman DA, Weiss DG, Bond JH, Ahnen DJ, Garewal H and Chejfec G: Use of colonoscopy to screen asymptomatic adults for colorectal cancer. N Engl J Med 343: 162-168, 2000.

22. Burt RW: Colon cancer screening. Gastroenterology 119: 837-853, 2000.

23. Ahmed FE, Ahmed NC, Gouda M and Vos P: MiRNAs for the diagnostic screening of early stages of colon cancer in stool or blood. Surg Case Rep Rev 1: 1-19, 2017.

24. Baffa R, Fassan M, Volinia S, O'Hara B, Liu CG, Palazzo JP, Gardiman M, Rugge M, Gomella LG, Croce CM and Rosenberg A: MicroRNA expression profiling of human metastatic cancers identifies cancer gene targets. J Pathol 219: 214-221, 2009.

25. Osaki M, Okada F and Ochiya T: miRNA therapy targeting cancer stem cells: A new paradigm for cancer treatment and prevention of tumor recurrence. Ther Deliv 6: 323-337, 2015.

26. Feng L, Ma H, Chang L, Zhou X, Wang N, Zhao L, Zuo J, Wang Y, Han J and Wang G: Role of microRNA-141 in colorectal cancer with lymph node metastasis. Exp Ther Med 12: 3405-3410, 2016.

27. Li F, Chen H, Huang Y, Zhang Q, Xue J, Liu Z and Zheng F: miR-34c plays a role of tumor suppressor in HEC-1-B cells by targeting E2F3 protein. Oncol Rep 33: 3069-3074, 2015.

28. Yang S, Li W, Sun H, Wu B, Ji F, Sun T, Chang H, Shen P, Wang Y and Zhou D: Resveratrol elicits anti-colorectal cancer effect by activating miR-34c-KITLG in vitro and in vivo. BMC Cancer 15: 969, 2015.

29. Gao Y, Feng B, Han S, Zhang K, Chen J, Li C, Wang R and Chen L: The roles of MicroRNA-141 in human cancers: From diagnosis to treatment. Cell Physiol Biochem 38: 427-448, 2016.

30. Tao F, Tian X, Lu M and Zhang Z: A novel lncRNA, Lnc-OC1, promotes ovarian cancer cell proliferation and migration by sponging miR-34a and miR-34c. J Genet Genomics 45: 137-145, 2018.

31. Wang J, Wang G, Li B, Qiu C and He M: miR-141-3p is a key negative regulator of the EGFR pathway in osteosarcoma. Onco Targets Ther 11: 4461, 2018.

This work is licensed under a Creative Commons Attribution-NonCommercial-NoDerivatives 4.0 International (CC BY-NC-ND 4.0) License. 